\title{
On Modeling Departure Time Choice for Home-Based Social/Recreational and Shopping Trips
}

\author{
Jennifer L. Steed and Chandra R. Bhat \\ Department of Civil Engineering, The University of Texas at Austin \\ ECJ 6.806, Austin, Texas 78712, Tel: (512) 471-4535, Fax: (512) 475-8744 \\ E-mail: jlsteed@mail.utexas.edu, bhat@mail.utexas.edu
}

\begin{abstract}
The existing literature on departure time choice has primarily focused on work trips. In this paper, we examine departure time choice for non-work trips, which constitute an increasingly large proportion of urban trips. Discrete choice models are estimated for home-based social/recreational and home-based shopping trips using the 1996 activity survey data collected in the Dallas-Fort Worth metropolitan area. The effects of individual and household socio-demographics, employment attributes, and trip characteristics on departure time choice are presented and discussed. The results indicate that departure time choice for social/recreational trips and shopping trips are determined for the most part by individual/household socio-demographics and employment characteristics, and to a lesser extent by trip level-of-service characteristics. This suggests that departure time for social/recreational and shopping trips are not as flexible as one might expect and are confined to certain times of day because of overall scheduling constraints. The paper concludes by identifying future methodological and empirical extensions of the current research.
\end{abstract}




\section{INTRODUCTION}

Several dimensions of choice characterize individuals' trip-making, including trip frequency, trip destination, travel mode, trip route and time-of-day of travel. Most metropolitan planning organizations (MPOs) include explicit components to model trip frequency, destination, mode and route as part of their trip-based travel demand analysis frameworks. On the other hand, the time-of-day dimension of travel has received little attention in the trip-based modeling approaches adopted by MPOs. The reason for this lack of attention to time-of-day may be traced back to the context in which the trip-based modeling framework was developed in the 1960s. The primary objective then was to evaluate alternative major capital improvements, so the focus was on predicting how alternative projects would affect overall daily travel demand levels and not on predicting shifts in travel within a day (1).

While evaluating capital improvements continues to remain an important objective of travel demand models, there has been a shift in emphasis in the past decade from evaluating long-term investment-based strategies to understanding travel behavior responses to shorter term, time-of-day specific, congestion management policies such as peak period pricing and peak period high occupancy vehicle use incentives. In addition, air quality modeling requires temporal resolution in the number of vehicle trips because a) the emissions factors (in grams per mile) to be applied to vehicle miles of travel (VMT) are sensitive to meteorological conditions (temperature and humidity) and vary considerably by time-ofday, b) the operating mode of trips are quite different across times of day (for example, a large proportion of trips in the morning and afternoon peak periods begin in the cold-start 
mode relative to other periods of the day), and c) photochemical dispersion models to determine ozone formation require mobile source emission levels of ozone precursor pollutants (i.e., Oxides of Nitrogen and Volatile Organic Compound) by time of day (see Chatterjee et al. (2)).

The recognition of the need for temporal resolution in trip-making has led MPOs of some large metropolitan areas to apply fixed, aggregate-level, factors to apportion the predicted total daily travel to different times of the day. The use of such fixed factors in travel modeling represents an improvement over disregarding the time-of-day dimension entirely. However, it is still very simplistic and inadequate for a number of reasons. First, fixed factors implicitly assume that trip departure times are unaffected by employment-related and socio-demographic characteristics. This is a rather untenable assumption since it is very likely that employment and socio-demographic attributes are associated with constraints/preferences regarding time-of-day of participation in activities. It is particularly critical to accommodate these effects at a time when there are substantial changes in employment and socio-demographic attributes of the population which can lead to trip timing patterns in the future that are very different from those existing today. Assuming that trip timing will remain the same in the future, therefore, can lead to inappropriate policy evaluations of congestion alleviation strategies and misinformed air quality plans (see Deakin, Harvey, Skabardonis, Inc. (3)). Second, applying aggregate-level factors to apportion trips to different times of the day does not accommodate departure time switching that might occur due to non-uniform (across time-of-day) variations in roadway conditions between the estimation and forecast years. 
Third, if time-of-day specific transportation control measures (such as congestion pricing or peak-period pricing) are implemented, the resulting temporal displacements of trips can be evaluated only by modeling level-of-service sensitivities in departure time decisions (4).

The preceding discussion of the importance of modeling departure time decisions of individuals motivates the research in this paper. Specifically, this paper examines the effect of individual and household socio-demographic characteristics, employmentrelated attributes, and trip characteristics on departure time choices of individuals. The departure time alternatives are represented by several temporally contiguous discrete time periods such as early morning, a.m. peak, a.m. off-peak, p.m. off-peak, p.m. peak, and evening. The choice among these alternatives is modeled using a discrete choice model.

Within the context of departure time choice, the focus of this study is on non-work trips. The reason for our emphasis on non-work trips is two-fold. First, non-work trips constitute an increasingly large proportion of urban trips and, therefore, have a significant impact on traffic congestion and air quality (see Gordon et al. (5)). Second, individuals are likely to have more temporal flexibility for pursuing non-work activities than they do for commuting to work. The implication of this greater flexibility for non-work trips is that socio-demographic changes and/or transportation control measures may have a more significant impact on such trips than on the less flexible work trips.

To narrow the scope of the research, we further examine departure time choice only for home-based social/recreational trips and shopping trips within the broad category of non-work trips. These two selected trip purposes comprise the largest proportion of non- 
work trips in most metropolitan areas (a home-based trip of any purpose refers to all trips which have that purpose at one trip end and home at the other trip end).

The existing literature on departure time choice has primarily focused on work trips (this is another reason for our study of non-work trips in this research). Examples of previous work departure time studies include Abkowitz (6), Small (7), Hendrickson and Plank (8), Mannering (9), Chin (10), Liu and Mahmassani (11), and Purvis (12). In contrast to the substantial number of work departure time studies, only a handful of studies have examined non-work departure time choice. We briefly review these studies below.

Kumar and Levinson (13) analyze the distribution and type of non-work trips during different times of the day. Their results suggest considerable variation in the temporal pattern of non-work trips between workers and non-workers, and between shopping and other non-work activities. The study is insightful, but is conducted at an aggregate descriptive level rather than at an individual choice level.

Hunt and Patterson (14) analyze recreational trip departure time choice at the individual choice level. Their study is conducted in the context of a hypothetical recreational trip to the movies. The choice of the movie start time is pre-determined, and the emphasis is on understanding departure time choice (from home) based on factors such as automobile travel time, desired "cushion" time at the theatre before the movie begins, the probability of being late, parking cost, and whether the movie is a new or old release. Since the movie start time is considered fixed in the study, there is limited 
temporal flexibility in departure time (as in the case of the work departure time studies reviewed earlier).

More recently, in two separate papers, Bhat $(4,15)$ demonstrates the application of new discrete choice formulations for joint travel mode and departure time choice modeling for non-work trips in the Bay area. The emphasis is on model formulation rather than on empirical specification analysis. In the current paper, we consider an exhaustive set of variables in departure time choice models for the Dallas-Fort Worth area, and also compare and contrast the estimation results between the shopping and social/recreational trip purposes.

The remainder of this paper is organized as follows. Section 2 discusses data assembly procedures. Section 3 provides an overview of the model structure used in the analysis and presents empirical results. Finally, Section 4 highlights the important findings from the study and identifies future research directions.

\section{DATA ASSEMBLY}

\subsection{Data Sources}

The primary data source used for this analysis is the 1996 activity survey conducted in the Dallas-Fort Worth metropolitan area by the North Central Texas Council of Governments (NCTCOG). This survey included an activity diary to be filled out by all members of the household. The activity diary collected information on all activities undertaken during the diary day. For non-travel activities, information on the activity 
type, start and end times of participation, and location was collected. For travel activities, information on the mode of travel used, costs incurred, and trip duration was collected. In addition, the survey elicited individual and household socio-demographic information.

The secondary data source for our analysis is a level-of-service (LOS) data file obtained from NCTCOG which provides information on times, costs and distances for travel between each pair of the 919 Transportation Analysis Process (TAP) zones in the Dallas-Fort Worth metropolitan planning area. The LOS data varies by travel mode (drive alone, shared-ride and transit) and by time of day (peak and off-peak).

\subsection{Sample Formation}

The process of developing the sample for analysis involved several steps. First, we converted the raw composite (travel and non-travel) activity file into a corresponding trip file. In doing so, we retained information on the type of activity pursued at, and the TAP zone identifier for, the origin and destination ends of each trip. The start and end times of each trip were also retained from the activity file.

Second, we collapsed the 36-category typology used in defining activity types in the original survey into a broader eight-category classification. These eight broad activity categories are home, work, school, personal business, community activities, social/recreational, shopping, and other (for ease in presentation, we will refer to the social/recreational category simply as the recreational category henceforth). The disaggregate activity categories classified as recreational activity include eating out, going to the gym or health club, exercise and recreation, entertainment, and visiting 
family/friends, while the disaggregate categories classified as shopping activity include both grocery and non-grocery shopping.

Third, we identified and selected those trips that were home-based recreational and home-based shopping trips using the activity type designations at each end of the trip. As indicated earlier, the analysis in this paper is confined to trips of these two purposes because they constitute a major fraction of total home-based non-work trips (in the Dallas-Fort Worth data, these two trip purposes together represent about $50 \%$ of all home-based non-work trips).

Fourth, we associated the departure time of each trip with one of the following six time periods of the day: early morning (midnight-6:30 a.m.), a.m. peak (6:30 a.m.-9:00 a.m.), a.m. off-peak (9:00 a.m.-noon), p.m. off-peak (noon-4:00 p.m.), p.m. peak (4:00 p.m.-6:30 p.m.), and evening (6:30 p.m.-midnight). The time periods for the a.m. and p.m. peaks were based on the peak periods definitions employed by NCTCOG in the Dallas-Fort Worth area. The times for the off-peak periods were determined by splitting the remaining blocks of time at noon and midnight. The dependent variable in our analysis is the choice of departure time among these six time periods.

$\underline{\text { Fifth, }}$ we matched the trip data with the appropriate socio-demographic characteristics of the individual pursuing the trip, and his household.

$\underline{\text { Sixth, }}$ we appended the appropriate LOS data to each trip record based on the origin/destination TAP zones and the mode used for the trip (almost all trips for shopping and for social-recreational activity are pursued using the drive alone, shared-ride or walk modes, and the analysis is restricted to trips using one of these modes; interestingly, there 
is not a single home-based shopping or recreational trip in the sample that uses public transportation). The drive alone and shared-ride LOS information was obtained from the TAP zone-level LOS data file provided by NCTCOG. The walk LOS information was computed from the distance between TAP zones and an assumed walk speed of 3 miles per hour.

Finally, we conducted several screening and consistency checks on the resulting data set from the previous steps (a flow chart of this screening process is available from the authors). As part of this screening process, we eliminated observations that had missing data on departure times and/or on the location of the origin/destination ends of the trip (the latter information is needed to obtain the level-of-service information for the trip). Several observations had missing income information and we imputed income for such observations based on a relationship between income and relevant socio-demographic attributes estimated from the sample of individuals who provided income information.

The final samples for analysis include 3178 observations for the home-based recreational trip category and 2056 observations for the home-based shopping trip category. These final samples represent approximately two-thirds of the total number of trips in the corresponding trip categories in the original raw data. The primary reason for the substantial reduction is the lack of origin/destination TAP zone data for many trips (because of which LOS information for these trips could not be determined). However, the observed split of trips among the six time periods is approximately the same in our final sample and in the original raw data. 


\subsection{Sample Description}

The distribution of departure times for recreational and shopping trips in the final samples are presented in Figure 1. For both trip categories, the number of trips increases as the day progresses. This trend is particularly noticeable for recreational trips. For shopping trips, there is little variation in the percentage of trips among the later periods of the day (i.e., the p.m. peak, p.m. off-peak and evening periods). As might be expected, very few trips of either type occur in the early morning or morning peak hours.

Table 1 shows the distribution of trips among the drive alone, shared-ride and walk modes. The dominant mode for recreational trips is shared-ride, indicating joint activity participation by several family members. The dominant mode for shopping is drive alone. This suggests less joint activity participation for shopping than for recreation. Overall, the splits between drive alone and shared ride are the reverse of one another between the recreational and shopping categories. Finally, recreational trips are more likely to be pursued using the walk mode compared to shopping trips.

An important note must be made here about travel mode choice. We consider mode choice as being exogenous to departure time choice. This decision is based on the observation that almost all recreational and shopping trips are pursued using a personal automobile (see Table 1). The distinction between drive alone and shared-ride modes is likely to be a reflection of how many individuals choose to participate jointly in the activity, not a conscious decision of which travel mode to use for the trip. We assume that the decision to engage in an activity with other individuals is made prior to the decision of what time to travel, and therefore consider travel mode choice between the 
drive alone and shared-ride modes as being pre-determined. Also, there is little variation in walk mode characteristics across different times of the day and hence it is quite reasonable to consider the choice of the walk mode to be exogenous to departure time choice. That is, individuals are likely to first make a decision to walk or not for a recreational/shopping activity, and then determine which time of day to pursue the activity.

\section{EMPIRICAL ANALYSIS}

\subsection{Model Structure}

The model structure used in this study is a discrete choice formulation among six time periods in a day, as indicated earlier in the paper. Discrete choice models are now commonly used in practice and a discrete departure time model can be relatively easily incorporated within the travel demand frameworks of MPOs. Our use of six time periods is more disaggregate than a simple peak versus off-peak breakdown and therefore better serves the purposes of evaluating congestion management policies and air quality modeling (see Section 1).

An alternative model structure for departure time modeling is a continuous-time duration representation (see Bhat (16) and Hensher and Mannering (17) for reviews of duration modeling methods). Such a continuous-time representation is appealing because it a) does not require the rather ad hoc partitioning of the day into time intervals, and b)

provides departure time of trips at a fine temporal resolution rather than in aggregate 
intervals. However, duration models have yet to find their way into practice. Besides, while continuous-time models have been used by researchers in the past for modeling departure time of trips, most of these studies have been confined to particular time periods of the day. For example, Hamed and Mannering (18) and Bhat (19) examine non-work trip departure time for workers after they arrive home from work at the evening, and Mannering and Hamed (20) study the length of time commuters delay their departure from work in the late afternoon to avoid peak period congestion. Within such time periods of the day, it may not be very unreasonable to assume that the effect of socio-demographic and employment characteristics do not change over time so that the familiar proportional hazard model (which assumes that the covariates change the baseline hazard by a constant factor that is independent of duration) may be applied. However, assuming fixed effects of socio-demographics and employment characteristics is untenable when the time domain for consideration is the entire day, as is the case in the current research. For example, the effect of children on the termination of the activity duration preceding participation in recreational activity may be much more "accelerated" during the evening than in earlier times of the day because the evening is most convenient (from schedule considerations) for joint activity participation with children. Such sudden non-monotonic accelerations (or decelerations) in the effect of variables over the course of the day cannot be captured by the traditional proportional hazard or accelerated lifetime models (the accelerated lifetime model allows time-varying effects, but specifies the time-varying effects to be monotonic and smooth in the time domain). Further, level-of-service variables change during the course of the day and one must 
accommodate these time-varying covariates within the duration model framework. Incorporating such time-varying coefficients and time-varying covariates in duration models poses an econometric challenge (specialized econometric software needs to be developed) and also presents specification and interpretational challenges (see Bhat (16)).

For the above reasons, and because of the widespread familiarity and use of discrete choice structures, we retain a discrete choice representation of departure time choice in this paper. Within the context of a discrete choice formulation, we explored two alternative structures. The first is the multinomial logit (MNL) structure and the second is an ordered generalized extreme value (OGEV) structure.

The MNL structure is appealing because it has a simple formulation. However, it is saddled with the Independence from Irrelevant Alternatives (IIA) property. In the context of departure time modeling, the IIA property implies that there is no increased degree of sensitivity (due to excluded exogenous factors) between adjacent departure time alternatives compared to non-adjacent departure time alternatives. Thus, for example, implementation of congestion pricing during the p.m. peak period will result in an equal proportionate increase in the probability of choice of the a.m. off-peak period and in the probability of choice of the p.m. off-peak period.

The OGEV structure generalizes the MNL structure by allowing an increased degree of sensitivity (due to excluded exogenous factors) between adjacent departure time alternatives compared to between non-adjacent departure time alternatives (the reader is referred to Small (21) or Bhat (4) for a mathematical description of the OGEV model). Thus, it is not limited by the IIA restriction. In this structure, implementation of 
congestion pricing in the p.m. peak will result in a larger proportionate increase in the p.m. off-peak choice probability relative to the a.m. off-peak choice probability.

In our estimations, we first developed a preferred model specification based on the MNL structure and then tested the OGEV structure with this preferred specification. For both recreational and shopping trip categories, our empirical results indicated that the MNL structure is adequate in representing departure time choice in terms of data fit. Also, the dissimilarity parameter in the OGEV model was greater than 1, implying inconsistency with utility-maximizing theory (for the recreational purpose, the dissimilarity parameter was 1.489 with a t-statistic of 1.18 for testing the parameter against the null of 1.0; for the shopping purpose, the corresponding parameter value and t-statistic were 1.451 and 0.91 , respectively). Hence, we chose the MNL structure in the current analysis.

\subsection{Variable Specification}

Several types of variables were considered in the recreational and shopping departure time choice models. These include individual socio-demographics, household sociodemographics, individual employment-related attributes, and trip-related characteristics.

Individual socio-demographic characteristics explored in our specifications included dummy variables for sex, ethnicity and education level, and continuous representations of income and age. Household socio-demographic characteristics considered in the model included household size, the number and age distribution of children, the number and employment status of household adults, and the continuous value of household income. 
Individual employment-related attributes included dummy variables for employed individuals, self-employed individuals, students, homemakers and retired persons, as well as a continuous variable indicating the number of hours worked per week. The final category of variables was the trip-related characteristics, including whether the trip was to/from the recreational/shopping activity, the mode used for the trip, the trip travel time and trip travel cost.

For many of the variables discussed above, we had strong a priori expectations regarding the direction of their impact on departure time choice. For example, employed individuals are unlikely to undertake shopping or recreational trips during the mid-day periods. Similarly, we anticipated that individuals whose households have very young children would be likely to avoid the evening periods because of the biological needs of young children toward the end of the day. However, for some of the variables we considered in our specification, we did not have strong a priori expectations about the directionality of their effect. We included these variables to explore their effects on temporal trip-making patterns using empirical data.

The final model specifications were determined by adopting a systematic process of introducing new variables to the market share model (i.e., the constants only model), eliminating statistically insignificant variables, and combining variables when their effects are not significantly different. This systematic statistical process was informed by intuitive considerations and parsimony in the representation of variable effects. 


\subsection{Empirical Results}

The final model specification results are presented in Table 2. Before discussing the detailed results, an important point to note is that the practice of applying fixed factors to apportion daily travel to various time periods is equivalent to a model specification with only constants (i.e., a market shares model). This restrictive model can be statistically tested with the model proposed in this paper using a standard likelihood ratio test; the restricted log-likelihood value is the market share log-likelihood value in Table 2 and the unrestricted log-likelihood value is the log-likelihood at convergence. The appropriate likelihood ratio test value is 1033.0 for the recreational purpose and 627.6 for the shopping purpose. These values far exceed the corresponding chi-squared values with 18 degrees of freedom for the recreational purpose and with 16 degrees of freedom for the shopping purpose, at any reasonable level of significance. Thus, the tests strongly reject the fixed factor models in favor of the models proposed in Table 2.

We now proceed to discussing the parameter results by variable group. For all alternative-specific variables (i.e., for all variables except the total time variable in Table 2), the evening time period is the base. In instances where only a few time periods appear for a variable, all the excluded time periods, along with the evening period, form the base.

\subsubsection{Individual Socio-Demographics}

Among the individual socio-demographic variables, the effect of age suggests that older individuals avoid the early morning and late evening periods. This may be a 
reflection of their physiological need for more time to start the day and a desire to arrive home early because of safety/security considerations. In addition to the linear effect presented in the table, we also explored non-linear spline effects of age; however, these non-linear effects did not dramatically improve data fit and were also difficult to interpret. An approach that assigned people to discrete categories based on age was also tested, but the linear specification of age as a continuous variable was preferred.

The only ethnicity variable that has a significant effect on departure time is a variable indicating if a person is of mixed race (other race variables, such as Asian, AfricanAmerican, and Native American were included in the model with the base race being Caucasian, but none were found to have a significant impact on departure time). The parameter on the mixed race variable indicates that individuals with such a family heritage are likely to pursue recreational activities during the mid-day periods. This result is difficult to explain, but is retained because of the rapid growth of people with mixed race in Texas. Ethnicity did not have any effect on departure time for shopping trips.

The final individual socio-demographic variable is income. For shopping trips, individuals with higher income have a lower preference for the a.m. peak and mid-day periods. This may be the result of tighter schedule constraints of high-income-earning individuals during the mid-day, though this effect does not appear to carry over to recreational activities. As with the age variable, spline effects were also tested to see if income has a non-linear effect on departure time. However, the spline representation did not improve the model significantly, and the results were difficult to interpret. 


\subsubsection{Household-Level Socio-Demographics}

Several household socio-demographic attributes were tested in our models, as indicated in Section 3.2. However, the only variables appearing in the final specifications are associated with the presence and age distribution of children. The results show that individuals whose households have young children (less than 5 years of age) are more likely to pursue recreational trips during the earlier periods of the day (early morning through p.m. off-peak) than in the p.m. peak or evening periods. This may be related to the biological needs of young children toward the end of the day. However, individuals whose households have children between the ages of 6 and 15 tend to pursue recreational trips during the p.m. peak. This is quite intuitive since the p.m. peak offers a convenient time for joint activity participation in recreational activities with school-going children.

The effect of young children for the shopping trip purpose suggests a lower likelihood of participation during the mid-day (a.m. and p.m. off-peak) hours. Perhaps this is a result of the tendency to shop alone by an adult who must remain at home in the mid-day to take care of the needs of young children. The presence of children between the ages of 6 and 15 suggests a greater likelihood of participation during the a.m. peak hours.

Overall, recreational activities are likely to be pursued jointly with children and so participation in such activities appears to be organized around the schedule availability of children (i.e., during the day for households with young children and in the p.m. peak for households with older children). However, shopping activities may be pursued alone and so these activities are scheduled in periods when young children have fewer biological 
needs or during times when older children are at school. For both trip purpose categories, the number of children in the household did not have any significant impact beyond that of the presence of children.

\subsubsection{Individual Employment-Related Attributes}

The individual employment-related attributes in the final model specification include the number of work hours per week, a dummy variable indicating if the individual is selfemployed, and another dummy variable indicating if the individual is a student.

The effect of the number of hours of work variable indicates that individuals who are employed and have a substantial work commitment are very unlikely to participate in recreational or shopping activities during the mid-day periods (i.e., the a.m. and p.m. offpeak periods). This is a reasonable result since such individuals would be at work during these times. These individuals are also less likely to participate in recreational/shopping activities in the peak periods (especially the a.m. peak) relative to the evening period. Overall, individuals who are employed and work many hours are likely to participate in recreational/shopping activities during the evening period because of work schedule constraints during the earlier times of the day (technically speaking, the results suggest that working individuals are as likely to participate in activities during the early morning period as in the evening period; however, this result is simply a statistical manifestation of the extremely few number of working individuals who choose the early morning period). 
Between the recreational and shopping trip purposes, employed individuals appear to be less likely to participate in shopping during the mid-day periods than to participate in recreation. This may be a result of two underlying factors. First, individuals may prefer to pursue shopping activities just prior to returning home rather than during the mid-day when they have to return to the office. Second, eating out is classified as a recreational activity in our analysis and employees are obviously more likely to eat out during the mid-day than shop. ${ }^{1}$

The effect of the self-employed variable within the category of individual employment-related attributes is interesting. Self-employed individuals are more likely to participate in recreational and shopping activities during the mid-day than externally (non-self) employed individuals. That is, self-employed individuals are able to "sandwich" a recreational/shopping activity from home (or wherever else their workplace is) between periods of a.m. and p.m. work because of lesser schedule rigidity. Between the two trip purposes, self-employed individuals are more likely to participate in shopping trips during the mid-day than in recreational trips.

${ }^{1}$ This point raises an important issue since eating out during the mid-day may be better characterized as a biological maintenance need rather than a recreational activity; however, eating out during the evening periods is more likely to be part of a social/recreational activity. Accommodating the differential nature of the same activity across time periods of the day would be useful, but would also make the travel demand forecasting process cumbersome. 
We considered a specification (for each trip purpose) that also included a dummy variable corresponding to external-employment in addition to the number of hours and self-employment variables. Such a variable would add a generic effect (i.e., independent of hours of work) of being externally employed. The resulting specification showed a marginal (though statistically significant) improvement in data fit, but also led to results that were quite difficult to interpret. Therefore, we excluded the external-employment dummy variable. An alternative specification that replaced the hours of work variable with the external-employment dummy variable also performed well and provided easyto-interpret results, but was statistically inferior to the current specification (however, this alternative specification might offer forecasting advantages since it only requires forecasting employment status, not hours of work).

The final variable under employment-related attributes is the student dummy variable. The coefficients on this variable suggest a preference by students for the p.m. peak and evening periods for participation in recreational/shopping activities. An interesting difference between students and employed individuals (externally-employed or selfemployed) is that students are equally likely to participate in recreational/shopping activities during the p.m. peak and evening periods, while employed individuals are more likely to choose the evening period than the p.m. peak. This difference may be attributed to the increased flexibility of students during the p.m. peak, since the typical school day of a student ends earlier than the typical workday of an employee. 


\subsubsection{Trip-Related Attributes}

Three trip-related attributes appear in the final model specification: a dummy variable indicating whether a trip is from home to a recreational/shopping activity or from the activity to home, another dummy variable indicating if the mode used for the trip is drive alone or not, and the trip travel time.

The results of the impact of the "home to activity" variable on recreational departure time suggest that trips from home to an activity are likely to be pursued before the evening periods, and especially in the a.m. peak period. This is expected, since most trips originating in the a.m. peak are likely to be leaving home. The same is true, though to a lesser extent, for the a.m. off-peak period. The increased likelihood of trips from home to a recreational activity in the p.m. peak compared to the morning and p.m. off-peak periods can be attributed to the temporal "resurgence" in recreational participation during the p.m. peak period; on the other hand, a sizable fraction of home-based recreational trips in the early morning period are late return home trips from the previous evening's recreational engagement and many p.m. off-peak home-based recreational trips are return home trips after recreational participation in the a.m. periods. The impact of the "home to activity" variable on shopping departure time is more straightforward; there is a strong preference to pursue shopping activities during the morning periods.

The drive-alone dummy variable effects in the table indicate a general trend toward the use of the drive alone mode during the earlier periods of the day (morning, a.m. peak and mid-day) and the use of non-drive alone modes (shared-ride and walking) during the later periods of the day (p.m. peak and evening). This is a rather intuitive result; trips 
during the day are more likely to be pursued alone, while the late afternoon and evening periods are times that are most convenient for joint activity participation and for walking.

The only statistically significant level-of-service attribute in the departure time choice model is trip travel time. Even this variable has a significant impact only for the recreational purpose. The negative effect is consistent with a priori expectations; individuals prefer departure times that result in shorter travel times. The lack of any significant effect of trip travel time on shopping departure time choice is interesting. Two related issues may be at work here. First, shopping activities are organized around work constraints and other household schedule considerations, and are pursued at the most convenient time within these schedule considerations. Travel time may therefore not play a substantial role in shopping departure time decisions. Second, shopping trips tend to be of shorter length than recreational trips. In the context of the shorter lengths of shopping trips, there is likely to be smaller variation in travel times across time periods (in our data, the travel time variation across time periods for shopping trips is lower than for recreational trips). This may be manifesting itself in the form of the lack of any travel time effect on shopping departure time decisions.

A travel cost variable was also considered in the analysis, but did not have a statistically significant impact. 


\section{CONCLUSIONS}

This paper presents models for home-based recreational and shopping departure time choice. The departure time alternatives are represented by six temporally contiguous discrete time periods that collectively span the entire day.

Two model structures are explored for departure time choice among the six discrete periods: the multinomial logit and the ordered generalized extreme value structure. The latter is a generalization of the former and allows an increased degree of sensitivity (due to excluded factors) between temporally adjacent departure periods compared to temporally non-adjacent periods. For both recreational and shopping trip purposes, our analysis indicates that the MNL model is adequate to model departure time choice.

The empirical analysis in the paper uses the 1996 activity survey data collected by the North Central Texas Council of Governments (NCTCOG) in the Dallas-Fort Worth area. Several sets of variables were considered in our model specifications, including individual and household socio-demographics, employment-related attributes and triprelated characteristics. Important overall results from our empirical analysis are as follows:

- older individuals are most likely to participate in recreational and shopping activities during the mid-day,

- high-income-earning individuals have the opposite tendency and avoid the mid-day periods,

- individuals with very young children (under 5 years) in their households are unlikely to pursue recreational activities during the p.m. peak and evening, presumably 
because of the increased biological needs of young children during these late times of the day,

- individuals with children above 5 years in their households, on the other hand, are most likely to pursue recreational activities during the p.m. peak period since this is the most convenient time to jointly participate in recreational activities,

- individuals with children below 5 years of age are unlikely to participate in shopping activities during the mid-day,

- employed individuals and students are most likely to participate in recreational/shopping activities during the latter parts of the day,

- self-employed individuals are more likely than externally employed individuals to "sandwich" a recreational/shopping activity between the a.m. and p.m. work periods,

- trips to a recreational/shopping activity from home tend to be made before the evening period,

- trips pursued together with others or by walk are likely to be undertaken during the evening periods, and

- in the current empirical context, the only level-of-service variable that has a significant impact is trip travel time and even this applies only for recreational trips.

The foregoing results have substantial implications for transportation-air quality analysis. Specifically, our analysis suggests that socio-demographic and employment related attributes have a significant impact on departure time for non-work trips. Ignoring these effects can lead to misinformed transportation planning and air quality 
decisions because of changing demographic and employment-related trends over the next few decades. For instance, $18 \%$ of the population will be 60 years or older in 2020 compared to about $13 \%$ today in the State of Texas, according to projections by the Texas State Data Center. Similarly, there is a rise in inter-racial marriages, and trends suggest a consequent steep rise in individuals with a mixed race heritage over the next few decades (22). The structure of the household is also changing rapidly with an increase in households with no children (projections suggest that households with no children below 18 years of age will increase from about $53 \%$ today to about $60 \%$ in the next decade (23)). The number of employed individuals in the household, and the number of selfemployed individuals, are on the rise (24) and this trend is likely to continue. All of these demographic and employment changes will have an effect on departure time choices, and the departure time model in this paper can be used to assess these impacts and provide reliable information regarding the temporal distribution of trips for input to transportation policy and air quality analysis. Of course, application of our model for forecasting would require regional socio-demographic and employment forecasts of ethnicity, household structure, and employment arrangements in addition to the age and income forecasts that are commonly used by MPOs. These additional forecasts may be obtained from supplementary data sources and our results suggest that it is important for MPOs to pursue such an effort (see also Deakin, Harvey, Skabardonis, Inc. (3) for the importance of including socio-demographic and lifestyle issues in forecasting travel behavior).

The need to include socio-demographic and employment attributes is not only important because of changes in such variables over time, but also because of spatial 
differences in these variables within a metropolitan region. Applying fixed factors to apportion total daily travel to different times of the day assumes away the existence of spatial demographic variations and will, in general, lead to incorrect network assignment volumes by time of day. This, in turn, can lead to inaccurate VMT and speed estimates by time of day and, consequently, inaccurate transportation-air quality analysis.

An interesting finding from our model is that level-of-service characteristics do not appear to substantially impact departure time for recreational and shopping trips. This has significant implications for time-of-day specific transportation control measures (such as peak-period pricing or converting a general-purpose lane to a high occupancy vehicle use lane). Specifically, our results imply that there will be little to no temporal displacements of recreational/shopping trips because of such policies. This suggests that recreational and shopping trips may not be as temporally flexible as one might think. That is, an individual considers participation in recreational/shopping activities within the larger spectrum of daily activities that need to be pursued based on her/his individual circumstances and household structure characteristics, and this narrows down the timeof-day of recreational/shopping pursuits. Thus, scheduling issues may be so overpowering in recreational/shopping participation that they render individuals insensitive to level-of-service changes. However, a couple of comments are in order here. First, the lack of sensitivity to level-of-service may be a result of little variation in times and costs across time periods in the sample. In other metropolitan areas where there are substantial time/cost differences across time periods (for example, because of congestion-pricing controls which are absent in the Dallas-Fort Worth area), the results 
may be different. A useful empirical extension of the current work, then, would be to estimate a model of departure time choice using a data set collected in an area where there are congestion-pricing or peak-period pricing control strategies. Second, the seeming lack of sensitivity to level-of-service may be attributable to the use of zone-tozone network data where the same times and costs are assigned to all trips between a particular zonal pair. Zone-to-zone impedance is considered an attribute of each individual trip, resulting in a confounding of individual heterogeneity (variations in impedance measures across trips between the same zonal pair) and place heterogeneity (variations in impedance measures across zonal pairs). This can lead to incorrectly estimated parameters on the level-of-service variables. An approach to handle this issue would be to use a multi-level cross-classified model of the type proposed recently by Bhat (25). Despite the cautionary notes above, there is a suggestion in our results that trips for shopping and recreation may not, after all, be as temporally flexible as widely perceived. Further empirical analysis focusing on this issue with other data sets would be valuable.

A possible methodological extension of the current work would involve the use of a continuous-time hazard duration model for analyzing departure time choice decisions, as discussed in Section 3.1. This approach would obviate the need for the rather ad hoc boundaries associated with classifying the day into discrete time periods. The authors are currently pursuing such an effort. 


\section{ACKNOWLEDGEMENTS}

This research was funded by the U.S. Department of Transportation through the Southwest Region University Transportation Center. The second author would also like to acknowledge funding support from the Texas Department of Transportation and the National Science Foundation grants DMS 9208758 and DMS 9313013 to the National Institute of Statistical Sciences (NISS). The constructive suggestions of four reviewers on an earlier draft of the paper are greatly appreciated. We are grateful to Mahmoud Ahmadi, Ken Cervenka, and Gustavo Baez of the North Central Texas Council of Governments for providing the data and assisting with data-related issues. 


\section{REFERENCES}

1. Cambridge Systematics, Inc. Short-Term Travel Model Improvements, Final Report. Prepared for the U.S. Department of Transportation and the U.S. Environmental Protection Agency, October 1994.

2. Chatterjee, A. et al. Improving Transportation Data for Mobile Source Emission Estimates. National Cooperative Highway Research Program Report 394, TRB, National Research Council, Washington, D.C., 1997.

3. Deakin, Harvey, Skabardonis, Inc. Manual of Regional Transportation Modeling Practice for Air Quality Analysis, The National Association of Regional Councils, Washington, D.C., 1993.

4. Bhat, Chandra R. Analysis of Travel Mode and Departure Time Choice for Urban Shopping Trips. Transportation Research B, Vol. 32, No. 6, August 1998, pp. 361371.

5. Gordon, Peter, Ajay Kumar, and Harry W. Richardson. Beyond the Journey to Work. Transportation Research A, Vol. 22, No. 6, November 1988, pp. 419-426.

6. Abkowitz, Mark D. An Analysis of the Commuter Departure Time Decision. Transportation, Vol. 10, 1981, pp. 283-297.

7. Small, Kenneth A. The Scheduling of Consumer Activities: Work Trips. The American Economic Review, Vol. 72, No. 3, June 1982, pp. 467-479.

8. Hendrickson, Chris, and Edward Plank. The Flexibility of Departure Times for Work Trips. Transportation Research A, Vol. 18, No. 1, January 1984, pp. 25-36. 
9. Mannering, Fred L. Poisson Analysis of Commuter Flexibility in Changing Routes and Departure Times. Transportation Research B, Vol. 23, No. 1, February 1989, pp. 53-60.

10. Chin, Anthony T. H. Influences on Commuter Trip Departure Time Decisions in Singapore. Transportation Research A, Vol. 24, No. 5, June 1990, pp. 321-333.

11. Liu, Yu-Hsin, and Hani S. Mahmassani. Dynamic Aspects of Departure Time and Route Decision Behavior Under Advanced Traveler Information Systems (ATIS): Modeling Framework and Experimental Results. Presented at the $77^{\text {th }}$ Annual Meeting of the Transportation Research Board, Washington, D.C., January 1998.

12. Purvis, Chuck. Peak Spreading Models: Promises and Limitations. Presented at the $7^{\text {th }}$ TRB Conference on Application of Transportation Planning Methods, Boston, MA, March 1999. http://www.mtc.ca.gov/datamart/research/boston2.htm.

13. Kumar, Ajay, and David Levinson. Temporal Variations on Allocation of Time. In Transportation Research Record 1493, TRB, National Research Council, Washington, D.C., 1995, pp. 118-127.

14. Hunt, J. D., and D. M. Patterson. A Stated Preference Examination of Time of Travel Choice for a Recreational Trip. Journal of Advanced Transportation, Vol. 30, No. 3 , 1996, pp. 17-44.

15. Bhat, Chandra R. Accommodating Flexible Substitution Patterns in MultiDimensional Choice Modeling: Formulation and Application to Travel Mode and Departure Time Choice. Transportation Research B, Vol. 32, No. 7, September 1998, pp. 455-466. 
16. Bhat, Chandra R. Duration Modeling, forthcoming in Handbook of Transport, Kenneth Button and David Hensher, eds, 1999.

17. Hensher, David A., and Fred L. Mannering. Hazard-Based Duration Models and their Application to Transport Analysis. Transport Reviews, Vol. 14, No. 1, 1994, pp. 63-82.

18. Hamed, Mohammad M. and Fred L. Mannering. Modeling Travelers' Postwork Activity Involvement: Toward a New Methodology. Transportation Science, Vol. 27, No. 4, November 1993, pp. 381-394.

19. Bhat, Chandra R. A Model of Post-Home Arrival Activity Participation Behavior. Transportation Research, Vol. 32B, No. 6, August 1998, pp. 387-400.

20. Mannering, Fred L. and Mohammed M. Hamed. Occurrence, Frequency, and Duration of Commuters' Work-to-Home Departure Delay. Transportation Research, Vol. 24B, No. 2, April 1990, pp. 99-109.

21. Small, Kenneth A. A Discrete Choice Model for Ordered Alternatives. Econometrica, Vol. 55, No. 2, pp. 409-424.

22. U.S. Census Bureau. Interracial Tables. Population Division, Racial Statistics Branch, 1998. http://www.census.gov/population/www/socdemo/interrace.html.

23. U.S. Census Bureau. Projected Number of Families with Children under 18 Years by Type: 1995 to 2010. Population Division, Population Projections Branch. http://www.census.gov/population/www/projections/nathh.html. 
24. U.S. Census Bureau. Working at Home. Population Division, Journey to Work and Migration Statistics Branch, 1999. http://www.census.gov/population/www/socdemo/workathome/wkhtab1.html

25. Bhat, Chandra R. A Multi-Level Cross-Classified Model for Discrete Response Variables. Forthcoming in Transportation Research, 1999. 


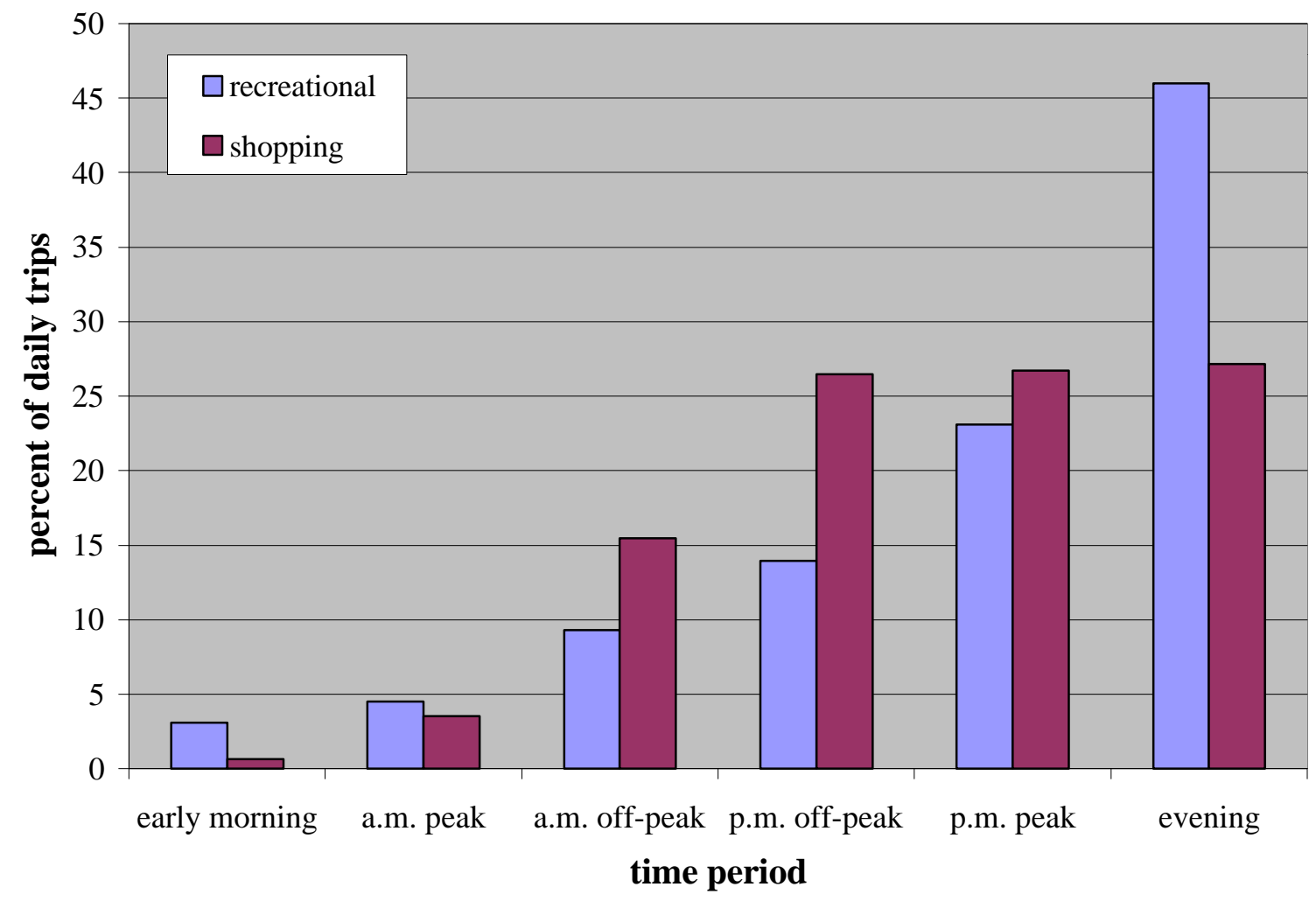

Figure 1. Time Distribution of Home-Based Recreational and Shopping Trips 
Table 1. Distribution of Modes Used for Recreational and Shopping Trips

\begin{tabular}{cccc}
\hline & \multicolumn{3}{c}{ Mode Used } \\
\cline { 2 - 4 } Trip Category & Drive Alone & Shared Ride & Walk \\
\hline Recreational & $33.7 \%$ & $59.9 \%$ & $6.4 \%$ \\
Shopping & $55.3 \%$ & $42.2 \%$ & $2.5 \%$ \\
\hline
\end{tabular}


Table 2. Recreational and Shopping Trip Departure Time Choice Models

\begin{tabular}{|c|c|c|c|c|}
\hline \multirow[b]{2}{*}{ Variables } & \multicolumn{2}{|c|}{ Recreational } & \multicolumn{2}{|c|}{ Shopping } \\
\hline & Parameter & t-statistic & Parameter & t-statistic \\
\hline \multicolumn{5}{|l|}{ Constant } \\
\hline morning & -3.761 & -23.29 & -5.059 & -13.88 \\
\hline a.m. peak & -4.320 & -15.63 & -2.804 & -8.69 \\
\hline a.m. off-peak & -2.949 & -14.39 & -1.028 & -4.25 \\
\hline p.m. off-peak & -2.132 & -11.22 & -0.320 & -1.38 \\
\hline p.m. peak & -1.133 & -10.24 & -0.401 & -2.56 \\
\hline \multicolumn{5}{|l|}{$\underline{\text { Individual socio-demographics }}$} \\
\hline \multicolumn{5}{|l|}{ Age } \\
\hline a.m. peak & 0.019 & 6.41 & - & - \\
\hline a.m. off-peak, p.m. off-peak & 0.019 & 6.41 & 0.028 & 6.76 \\
\hline p.m. peak & - & - & 0.020 & 5.80 \\
\hline \multicolumn{5}{|l|}{ Ethnicity - mixed race } \\
\hline a.m. off-peak, p.m. off-peak & 0.928 & 3.87 & - & - \\
\hline \multicolumn{5}{|l|}{ Income (thousands) } \\
\hline a.m. peak, a.m. off-peak, p.m. off-peak & - & - & -0.053 & -2.51 \\
\hline \multicolumn{5}{|l|}{ Household-level socio-demographics } \\
\hline \multicolumn{5}{|l|}{$\overline{\text { Presence of children age } 5 \text { or under }}$} \\
\hline morning, a.m. peak & 0.957 & 7.89 & - & - \\
\hline a.m. off-peak, p.m. off-peak & 0.957 & 7.89 & -0.395 & -2.34 \\
\hline \multicolumn{5}{|l|}{ Presence of children age 6 to 15} \\
\hline a.m. peak & - & - & 0.637 & 2.47 \\
\hline p.m. peak & 0.339 & 3.57 & - & - \\
\hline \multicolumn{5}{|l|}{ Individual employment-related attributes } \\
\hline \multicolumn{5}{|l|}{ Hours worked per week } \\
\hline a.m. peak & -0.029 & -6.35 & -0.022 & -3.55 \\
\hline a.m. off-peak & -0.049 & -12.09 & -0.057 & -12.17 \\
\hline p.m. off-peak & -0.041 & -12.69 & -0.049 & -12.93 \\
\hline p.m. peak & -0.016 & -6.76 & -0.016 & -5.67 \\
\hline \multicolumn{5}{|l|}{ Self-employed } \\
\hline a.m. off-peak, p.m. off-peak & 0.997 & 6.19 & 0.719 & 3.47 \\
\hline \multicolumn{5}{|l|}{ Student } \\
\hline a.m. peak, a.m. off-peak & -0.753 & -5.14 & -1.196 & -4.85 \\
\hline p.m. off-peak & -0.753 & -5.14 & -0.962 & -4.33 \\
\hline \multicolumn{5}{|l|}{ Trip-related attributes } \\
\hline \multicolumn{5}{|l|}{ Home to activity } \\
\hline morning & 0.851 & 4.05 & 1.255 & 5.37 \\
\hline a.m. peak & 2.381 & 10.34 & 1.255 & 5.37 \\
\hline a.m. off-peak & 1.491 & 10.48 & 0.575 & 4.49 \\
\hline p.m. off-peak & 0.606 & 5.11 & - & - \\
\hline p.m. peak & 1.328 & 13.75 & - & - \\
\hline \multicolumn{5}{|l|}{ Drive alone mode } \\
\hline morning, a.m. peak & 1.262 & 12.26 & 1.121 & 4.69 \\
\hline a.m. off-peak, p.m. off-peak & 1.262 & 12.26 & 0.449 & 4.06 \\
\hline p.m. peak & 0.349 & 3.08 & - & - \\
\hline Total travel time & -0.022 & -2.36 & - & - \\
\hline \multicolumn{5}{|l|}{ Log Likelihoods } \\
\hline Equal Shares & \multicolumn{2}{|c|}{-5694.212} & \multicolumn{2}{|c|}{-3683.858} \\
\hline Market Shares & -457 & 521 & -3082 & 983 \\
\hline Convergence & -406 & & -2769 & \\
\hline Number of Observations & 31 & & 20 & \\
\hline
\end{tabular}




\section{LIST OF FIGURES AND TABLES}

Figure 1. Time Distribution of Home-Based Recreational and Shopping Trips

Table 1. Distribution of Modes Used for Recreational and Shopping Trips

Table 2. Recreational and Shopping Trip Departure Time Choice Model 